\title{
Fomento del Comercio Urbano. Experiencias en materia de dinamización en centros urbanos de barrios y ciudades medias del área de Barcelona
}

\author{
Enric Llarch * y Jordi Matheu **
}

\section{Antecedentes}

La Diputació de Barcelona, como institución de la Administración Local de segundo nivel, tiene entre sus principales objetivos el de prestar apoyo técnico y financiero a los Ayuntamientos en el desarrollo de las actividades que les son propias. En concreto, en el año 1995 se creó el Àrea de Promoció Econòmica, para dar respuesta a la creciente preocupación de los municipios por la problemática del paro y la necesidad de participar, desde el ámbito local, en la creación de actividad económica, como principal instrumento para combatir la desocupación entre los habitantes del municipio.

El comercio urbano, el comercio a pie de calle, es una de las principales actividades económicas en muchos de los municipios de Cataluña. Las aceleradas transformaciones sufridas por el sector comercial en los últimos años y el impacto que generaban en la vitalidad de los centros urbanos de nuestras ciudades impulsaron la realización de un estudio que efectuase una primera aproximación a la situación del comercio urbano que incluía una evaluación de 17 experiencias que con diverso nivel de maduración y de éxito se habían llevado a cabo para fomentar el comercio urbano. Este estudio permitió realizar un diagnóstico de la situación, identificar los elementos de éxito y de fracaso de las distintas experiencias de promoción del comercio urbano y formular líneas de actuación de cara a potenciar con el mayor éxito posible una actividad económica fundamentalmente endógena.

El resultado del trabajo, un extracto del cual ha sido publicado por la Diputació de Barcelona ${ }^{1}$, plantea la impulsión de proyectos de dinamización de los centros urbanos o históricos, de forma que en 1998 se iniciaron dos primeras experiencias piloto.

La formulación de nuevas líneas de actuación desde la Diputació de Barcelona en el ámbito del apoyo al comercio urbano ha coincidido con una creciente sensibilidad y voluntad de convertirse en un agente activo en este ámbito por parte de los Ayuntamientos. Todo ello ha conllevado que los Ayuntamientos se doten de estructuras específicas de apoyo al comercio urbano, especialmente a partir de las elecciones municipales de 1999, cuando se crean nuevas Concejalias de Comercio o se incluye la denominación de Comercio en las de Promoción Económica en la mayoría de las principales ciudades de la provincia de Barcelona.

En este artículo presentamos el resultado de estos cuatro años de trabajo, en los que han permitido avanzar con notables resultados en la formulación y la implementación de una línea de actuación para la promoción de los Centros Comerciales Urbanos. De esta forma, los iniciales proyectos de dinamización comercial del centro urbano se han ido completando con otras iniciativas en materia de modernización de mercados munici- 
pales, ordenación de los equipamientos comerciales y otros estudios y actuaciones específicas.

\section{El conjunto del proyecto de la Diputació}

La Diputació de Barcelona, en el marco de las actuaciones de fomento del comercio local y el comercio urbano, dispone de líneas que complementan el eje central de actuación que constituye la dinamización de centros urbanos

Los Mercados Municipales, por su carácter de equipamiento comercial público, constituyen una de las líneas de actuación que se está potenciando, a través de proyectos de modernización funcional, arquitectónica y de gestión. El objetivo es convertir los mercados municipales en centros comerciales modernos y competitivos que puedan desarrollar con eficacia las funciones de locomotora comercial en los centros urbanos y de garantizar que los ciudadanos puedan escoger libremente los formatos comerciales donde desean proveerse.

La Diputació también presta su apoyo económico y técnico a los municipios que, acogiéndose a las previsiones de la Ley de Comercio de la Generalitat de Catalunya, deseen dotarse de Programas de Orientación de los Establecimientos Comerciales (POEC). Los POECs son instrumentos de planificación comercial, con los que se pretende incorporar a la normativa urbanística y de ordenanzas municipales los criterios necesarios para garantizar el desarrollo de un comercio acorde con el modelo de ciudad deseado y que, en cualquier caso, asegure la dotación de oferta comercial adecuada a todas las zonas del municipio, defina polaridades y jerarquías de ejes y zonas comerciales, asegure la cobertura de las necesidades de accesibilidad - viales, aparcamientos- y genere un entorno amigable al ciudadano - peatonalización, señalización comercial, mobiliario urbano, oferta de servicios complementarios a los estrictamente comerciales...-

También se realizan actuaciones específicas, relacionadas con el comercio, pero no integradas en las lineas anteriores y se da ayudas financieras a la realización de Ferias Comerciales Locales.

En la Tabla 1 se puede observar la distribución del presupuesto por tipologías de acciones y el número de actuaciones impulsadas en el año 2000.

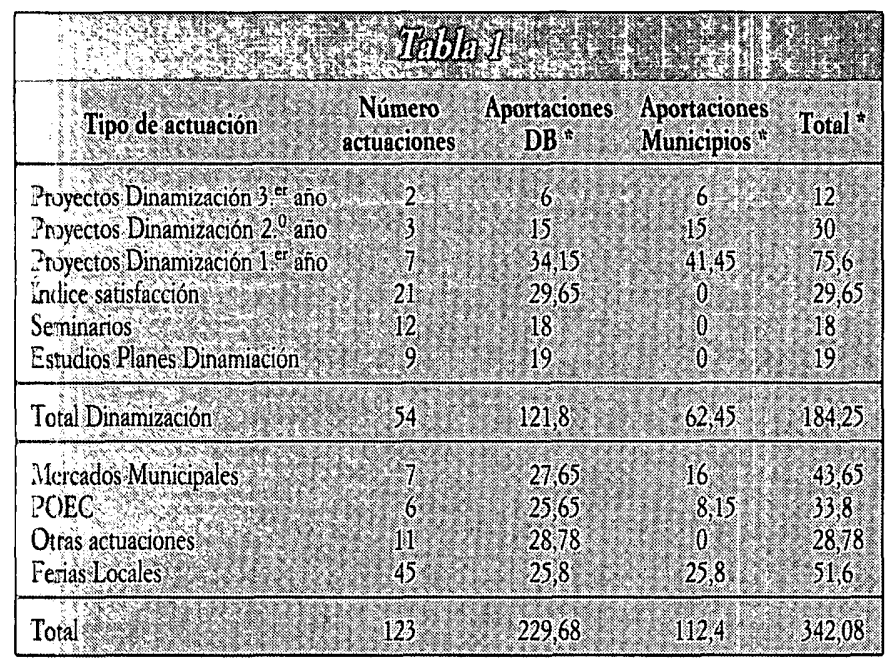

\# En 65 municipios.

* Millones de pesetas.

\section{La importancia del comercio urbano para el desarrollo del municipio}

Cada Ayuntamiento es responsable de planificar el desarrollo urbanístico de su municipio y de promover un modelo de ciudad que sea adecuado para las actividades económicas y las necesidades sociales de sus ciudadanos y que sea acorde con las aspiraciones individuales y colectivas de éstos.

Como administración pública más próxima a las personas, el Ayuntamiento ha de ser capaz de generar procesos de participación, comunicación y debate en torno al modelo de ciudad que se desea. Ha de fomentar un desarrollo cohesionado y sostenible, ser capaz de combinar la actividad económica, el ocio, la vivienda, la cultura, el respeto a las tradiciones y las relaciones sociales para poder satisfacer las necesidades de los ciudadanos del municipio. Este desarrollo, al mismo tiempo, debe prever y encauzar las futuras transformaciones de la ciudad de forma que no generen disfunciones que propicien, por ejemplo, que sus habitantes busquen satisfacer sus necesidades de trabajo, vivienda, ocio o de relación en otros territorios.

El requisito inicial e ineludible para implementar un programa de dinamización comercial es que las autoridades municipales deben asumir la necesidad de promocionar un centro urbano dinámico y activo, que refuerce su carácter de punto de referencia - tanto para sus habitantes tradicionales, como para los visitantes-, que facilite los intercambios y las relaciones - tanto económicas como sociales- entre todos los ciudadanos y que, al mismo tiempo, proyecte la ciudad hacia el exterior y le permita ser competitiva con otros territorios para satisfacer las necesidades de los ciudadanos y atraer más usuarios a los servicios, públicos y privados, disponibles.

Para el Ayuntamiento, la potenciación del centro es una actuación estratégica que genera dinámicas transformadoras sobre su entorno, que puede abordar simultáneamente objetivos de competitividad y de cohesión social, por la combinación que se genera entre el papel impulsor o regulador del sector público y la participación de diversos actores privados en su desarrollo, porque son susceptibles de promover un salto de calidad en la ciudad.

Pero a la vez, como actuación estratégica, la dinamización del centro urbano ha de estar integrada en una política de conjunto coherente, que se proponga al mismo tiempo elevar la escala de la ciudad y articular la existente. Esta actuación global precisa de instrumentos, como un Plan de Actuación de dinamización del centro urbano, un programa de actuaciones concertado a un horizonte fijado, planes sectoriales que integren diversas dimensiones como transporte, circulación, medio ambiente urbano, etcétera.

\section{Comercio y Administración local}

a) Responsabilidad Institucional

a.1) Mandato legal de asegurar la provisión de productos a través de los mercados municipales.

a.2) Garantizar el acceso de los ciudadanos a una oferta comercial adecuada y la efectiva capacidad de elección entre los diversos formatos comerciales. 
b) Ocupación y comercio minorista

b.1) Actividad intensiva en puestos de trabajo.

b.2) Nuevos servicios al consumidor: Yacimientos de ocupación.

c) Estructura productiva local y comercio urbano

c.1) Principal actividad económica en muchos municipios.

c.2) Flexibilidad, adaptación al medio y actividad endógena.

d) Modelo europeo de ciudad

d.1) Centralidad.

d.2) Diversidad.

d.3) Acumulación de patrimonio.

e) La ciudad, principal entomo para las relaciones

e.1) Humanas.

e.2) Económicas.

e.3) Sociales.

f) El comercio urbano da vida a los centros urbanos

f.1) Capacidad de atracción.

f.2) Fomento de las relaciones.

g) Incidencia del comercio urbano en la dinámica del municipio

g.1) Factores tangibles:

- Retención de la capacidad de consumo local.

- Captación de consumo externo.

- Generación de actividad económica.

- Captación de inversiones externas.

- Sinergias sociales, comerciales, culturales y lúdicas.

g.2) Factores intangibles:

- Cohesión territorial y social.

- Proyección exterior de la ciudad.

- Identificación de nuevos residentes con el municipio.

Pero si el Ayuntamiento es el responsable de los servicios comunes -accesibilidad, limpieza, mantenimiento, seguridad- el otro operador imprescindible en el proyecto de dinamización del centro comercial urbano es, por definición, el mismo establecimiento detallista. Por tanto, es imprescindible generar un entorno de confianza y de complicidades mutuas y establecer unos mecanismos de participación y de corresponsabilización que favorezcan que el proyecto sea asumido como propio, tanto por parte del Ayuntamiento como por el conjunto de los comerciantes.

Para ello, en la gran mayoría de casos, es necesario un cambio profundo en las relaciones entre el Ayuntamiento y el comercio local. La Administración Local debe reconocer de forma fehaciente la importancia del comercio en el funcionamiento del centro urbano, su papel relacional entre el centro urbano y el resto del municipio y la aportación a favor de la cohesión y la identidad local. Pero por parte de los comerciantes, también es necesario un esfuerzo para mentalizarse de que su negocio no termina en la puerta de la calle, que la cooperación entre ellos y con el resto de la ciudad es imprescindible para competir con éxito con los grandes y nuevos distribuidores comerciales periféricos y que la rentabilidad de su negocio depende en buena medida del dinamismo y de la calidad de los servicios que presta el centro urbano entendido como una gran área comercial a cielo abierto.

\section{Objetivos del proyecto de dinamización}

Concretar el objetivo general de un proyecto de dinamización del centro comercial urbano es complejo, dada la importancia que tienen los objetivos operativos que se incluyen. Para facilitar esta concreción, en primer lugar es clarificador resaltar lo que no es. No es un programa sectorial de promoción de la actividad comercial, ni una reforma urbanística de los centros de ciudad, ni un programa para combatir contra los nuevos hábitos de compra y preferencias de los ciudadanos.

Por el contrario, se pretende que la vida en las ciudades sea enriquecedora, fomente las relaciones humanas, que las ciudades se conviertan en un lugar agradable para vivir y desarrollarse económica y socialmente, en la que las necesidades de las personas queden cubiertas y en las que el ocio, la cultura y la actividad económica puedan convivir y complementarse mutuamente.

El objetivo general de un proyecto de dinamización del centro comercial urbano sería:

Fomentar la capacidad de atracción del centro unbano en materia comercial y de prestación de servicios como requisito indispen.

säble para que ejerza de punto de referencia, de intercambio

y de vertebración de la ciudad y sus habitantes.

Para concretar una propuesta tan genérica, aunque necesaria para remarcar la importancia de la globalidad del proyecto, es necesario detallar unos objetivos operativos de éste:

a) Potenciar la atractividad del centro urbano, con un mejor servicio a los usuarios, dando respuesta a las nuevas expectativas de los.consumidores en relación con la diversidad de la oferta comercial y la consideración de la compra como un acto lúdico, familiar y amigable. Albergando un amplio abanico de empresas comerciales, de ocio y de servicios complementarios competitivas y con carácter innovador, y también con una mejor imagen de la ciudad.

b) Promocionar y dar valor al centro de la ciudad con un entorno para vivir y comprar, para la cultura y el turismo, para invertir y desarrollar actividades de comunicación y animación. Potenciar la centralidad y la accesibilidad, el carácter simbólico y el entorno de calidad.

c) Atraer nuevas inversiones con una política urbanística de renovación y rehabilitación para un desarrollo de la actividad en un entorno y una calidad renovados.

d) Adoptar técnicas de organización y funcionamiento de los centros comerciales de nueva generación, teniendo en cuenta las especificidades propias de un centro urbano. 
La presentación de estos objetivos operativos permite visualizar con más claridad cuáles serán los contenidos del proyecto de dinamización del centro urbano que propone la Diputació de Barcelona. Sin embargo, antes de entrar en los contenidos y actuaciones del proyecto, es necesario relacionar o concretar estos objetivos con los diferentes actores que participan, fundamentalmente Ayuntamiento y Comerciantes del centro urbano.

Para el Ayuntamiento, el objetivo del proyecto se centra en la mejora de la gestión de un elemento clave para el modelo de ciudad como es el Centro Urbano, los aspectos del proyecto relacionados con las funciones que ha de desarrollar el centro en relación con el conjunto de la ciudad, con el fomento de la participación de los operadores económicos en el diseño, mantenimiento y gestión del centro urbano y la necesaria transversalidad del proyecto debido a la incidencia de actividades dependientes de diferentes áreas, como pueden ser la limpieza, la circulación, la planificación urbanística, la seguridad, el mobiliario urbano, la gestión de carga y descarga, la animación cultural, las actividades turísticas, etcétera.

Por tanto, para el Ayuntamiento los objetivos operativos se podrían enunciar mejor en términos de articulación de la relación del centro con el conjunto de la ciudad, de participación y de transversalidad.

Por parte de los comerciantes en su conjunto, el proyecto se centra en términos de competitividad. El centro comercial urbano ha de ser capaz de competir con éxito con los centros comerciales de nueva generación y las áreas comerciales de atracción externa. Es decir, para los comerciantes, los objetivos operativos están centrados en la capacidad de retener a los residentes en el municipio para satisfacer sus necesidades lúdicas y comerciales y al mismo tiempo en la capacidad de atracción del centro comercial urbano de residentes de otras localidades de la zona de influencia.

De todas formas, un objetivo operativo fundamental para la gestión del proyecto es la representatividad del conjunto de los comerciantes, la necesidad de disponer de un interlocutor válido que represente los intereses del conjunto de los operadores económicos del centro urbano, y que al mismo tiempo ejerza un liderazgo sobre el colectivo, que sea capaz de acercar al máximo las posiciones en torno al proyecto, es un elemento clave fundamental para el éxito de un proyecto de dinamización del centro comercial urbano.

\section{La propuesta metodológica}

1. Prioridades:

a) El punto de partida para los proyectos de dinamización impulsados por la Diputació es la implicación del Ayuntamiento, hasta el punto de liderarlo en su fase inicial, de un mínimo tres años. Sin la participación activa del principal gestor del entorno del centro urbano los resultados de la dinamización sólo podrán ser parciales.

b) La persuasión, la demostración con resultados, la incorporación gradual de los interlocutores individuales al proyecto, la implicación progresiva en el proyecto y el aumento de responsabilidades en la gestión, es la dinámica de relación que se propone entre los impulsores del proyecto, el área de Comercio del Ayuntamiento, y los operadores relacionados. Con esta dinámica se pretende conseguir en un plazo de tres años un colectivo suficiente de comerciantes identificados con el proyecto de dinamización del centro urbano, capaces de liderarlo y la participación de un mínimo del $70 \%$ de los comerciantes en las iniciativas que se impulsen.

c) La profesionalización de la gestión, para superar las limitaciones de los métodos tradicionales basados en el voluntarismo, a través de la figura del Gerente del Centro Comercial Urbano, es clave para el éxito del proyecto. Inicialmente es el Ayuntamiento el que asume su contratación, para asegurar una mínima estabilidad, una remuneración suficiente y una infraestructura de apoyo al desarrollo de sus actividades. Pero por los mismos argumentos que en el apartado anterior, el objetivo es que los comerciantes asuman la necesidad de esta gestión profesionalizada, de forma que en el plazo máximo de los tres años que dura el proyecto estén en disposición de garantizar económicamente la contratación del Gerente.

d) Fomentar la cultura colectiva, de cooperación empresarial entre operadores y de concertación publico-privada entre el conjunto de los operadores y el Ayuntamiento, impulsando una gestión conjunta del Centro Comercial Urbano para conseguir un centro comercial organizado.

e) Las aportaciones económicas de los operadores del Centro Comercial Urbano inicialmente son finalistas e individualizadas, dirigidas a colaborar en la financiación de actuaciones de captación y fidelización de la clientela, como campañas, promociones, servicios... El objetivo es minimizar el impacto de los free-riders: el comerciante que participe económicamente en una actuación de promoción o en la prestación de un servicio al cliente debe obtener una contrapartida directa e individualizada, que le permita diferenciarse frente al cliente de aquel otro comerciante que no participa.

2. Líneas de actuación del Proyecto de dinamización del Centro Comercial Urbano:

\subsection{Previas:}

a) Solicitud del Ayuntamiento a la Diputació de Barcelona para colaborar en un proyecto de dinamización.

b) Convenio de colaboración entre el Ayuntamiento y la Diputació de Barcelona, en el que se acuerdan las líneas de actuación del proyecto y las aportaciones anuales de las dos Instituciones, que siempre serán al $50 \%$, con un importe que 
oscilará entre los 24 y los 36 millones de pesetas a repartir en tres años.

c) Presentación del proyecto a los comerciantes más representativos del Centro Comercial Urbano.

\subsection{Inicio del proyecto:}

a) Delimitación espacial del Centro Comercial Urbano. Identificación con señalización efímera.

b) Creación del logo y del nombre comercial identificativo, realizando el registro de la marca por parte del Ayuntamiento.

c) Incorporación de la figura del Gerente como gestor profesional del Centro Comercial Urbano, con experiencia profesional suficiente, habilidades de liderazgo, organización y comunicación y conocimiento del entorno.

d) Elaboración de una propuesta de Plan de Actuación, en el que se especifiquen el calendario y el presupuesto, como mínimo del primer año.

\subsection{Contenidos del Plan de Actuación:}

a) Presentación del Proyecto: A comerciantes, de forma pública o restringida, exclusivamente a los asociados o incluyendo a los comerciantes más dinámicos, dependiendo de la situación del asociacionismo en cada localidad. A los medios de comunicación locales. A los Concejales y responsables técnicos de las áreas del Ayuntamiento más implicadas en los aspectos de gestión del Centro Urbano (limpieza, seguridad, urbanismo, vialidad, cultura, mantenimiento...).

\section{b) Actuaciones de promoción:}

- Son las que más fácilmente incorporan la necesidad de actuación conjunta entre los comerciantes, es más fácil conseguir su participación en la financiación de los costes y también conseguir demostrar la necesidad de un profesional que las organice y garantice el éxito.

- Son fundamentales para captar operadores que participen y que, en el futuro, se puedan asociar al proyecto de dinamización.

- La primera actuación de promoción: la campaña de Navidad. Hay que realizar actuaciones de promoción en el momento de máximo consumo y de mayor predisposición de los comerciantes para realizar una primera actuación conjunta. La campaña debe generàr un primer mensaje hacia el cliente de centro comercial urbano entendido como conjunto y contribuir a fidelizar a los clientes.

- Se consigue una aportación de los comerciantes finalista e individualizada, que relaciona directamente el esfuerzo económico del operador con el beneficio individual. El que no participa es fácilmente identificado por el cliente del centro comercial.

\section{c) Mejora de los servicios prestados a los clientes:}

- Proporcionar aparcamiento gratuito. Convenios con los aparcamientos ubicados en el Centro Urbano, campañas de difusión entre los clientes y de presentación individualizada a los comerciantes; asunción de los comerciantes del coste del tiquet entregado y utilizado.

- Tarjetas de fidelización de clientes. Distribución de puntos con diferentes tipologías de premios. Con posibilidad de migrar, a medio plazo, hacia una tarjeta de pago propia.

- Guarderías para los niños - para fomentar la compra familiar - organizadas por el Gerente del Centro Comercial Urbano, gestionadas directamente o por alguna organización especializada y con los costes directos del servicio financiadas por los comerciantes.

- Experiencias piloto de servicio a domicilio, combinado con sistemas de consigna.

d) Cooperación entre operadores comerciales:

- Acordar horarios conjuntos y días festivos de apertura, difundiendo el acuerdo como propio del Centro Comercial.

- Negociación conjunta con entidades financieras, mejoras en los costes por el cobro con tarjetas, aportaciones al proyecto de dinamización y otras ofertas de servicios financieros.

- Negociación con medios de comunicación para inserción de publicidad conjunta, del Centro Comercial Urbano y de los operadores interesados, consiguiendo una reducción de costes significativa y un impacto en los medios mayor.

- En general, actuar como central de compras para conseguir reducir costes y ajustar precios con el objeto de ser más competitivos. Las posibilidades son numerosas y abarcan tanto las compras como los suministros: bolsas de papel, servicios de telefonía, asesorías fiscales, laborales o financieras, seguros, distribución y almacenamiento...

e) Cooperación entre el Ayuntamiento y los interlocutores de los operadores comerciales y de servicios.

- Establecer un canal de comunicación estable, del Gerente con los representantes de los operadores y con un interlocutor responsable del proyecto del Ayuntamiento.

- Organizar comisiones técnicas por temas, como limpieza, seguridad o vialidad, en los que periódicamente se haga un seguimiento de las actuaciones y se analicen propuestas futuras.

- Una Comisión de Seguimiento que propone las principales líneas de actuación y analiza los resultados obtenidos, en la que participan todos los operadores y las instituciones implicadas en el proyecto.

f) Medidas para estructurar el Centro Urbano como un Centro Comercial.

- Señalización estable, a partir de un modelo para los Centros Comerciales Urbanos propuesto por la Direcció General de Comerç de la Generalitat de Catalunya.

- Organización periódica de actos de animación, coordinadas con el Ayuntamiento y las entidades culturales del municipio. 
- Colaboración entre comerciantes y cines, teatros, cafeterías y centros lúdicos en general, para actividades de promoción y animación del Centro Comercial Urbano.

2.4. Instrumentos incorporados a los proyectos de dinamización:

a) De seguimiento: Índice de Satisfacción del usuario del Centro Comercial Urbano. 400 encuestas realizadas en horario y días comerciales, que se repite anualmente y con la misma metodología en todos los municipios en los que se impulsan proyectos de dinamización. (Véase despiece adjunto.)

\section{Índice de Satisfacción de los usuarios del Centro Comercial Urbano}

Encuestas a 400 usuarios de los Centros Comerciales Urbanos (CCU), en horario comercial, los sábados y días de mercado ambulante.

Sobre un total de 17 factores relacionados con la actividad del centro comercial urbano -12 de comunes para todos los municipios y cinco más de específicos según las características y los intereses de cada uno de ellos-, se pregunta: CCU.

La Importancia: Nos indica lo que el usuario desea encontrar cuando se dirige al

La Valoración: Nos indica la apreciación por parte del usuario de lo que realmente encuentra.

Se calcula la diferencia entre importancia y valoración (GAP), que nos indica el grado de satisfacción del usuario del CCU para cada uno de los factores.

Con los resultados de Importancia y Valoración de cada uno de los factores se obtiene un Índice General Ponderado, que es una medida del funcionamiento de un Centro Comercial Urbano.

Se adjuntan los datos agregados de las encuestas realizadas en noviembre de 1999 en seis municipios del entorno urbano de Barcelona. En la Tabla adjunta se representan los datos de los 12 factores comunes ordenados por la Valoración. Destacan como factores más importantes la Calidad, el Precio, la Atención al cliente y la Limpieza, mientras que los más valorados son la Variedad de la oferta de productos, la Calidad, los Horarios y la Atención al cliente. Por contra, destaca la Restauración como el factor de menor importancia y el Aparcamiento y las Actividades lúdicas y culturales, como los factores menos valorados.

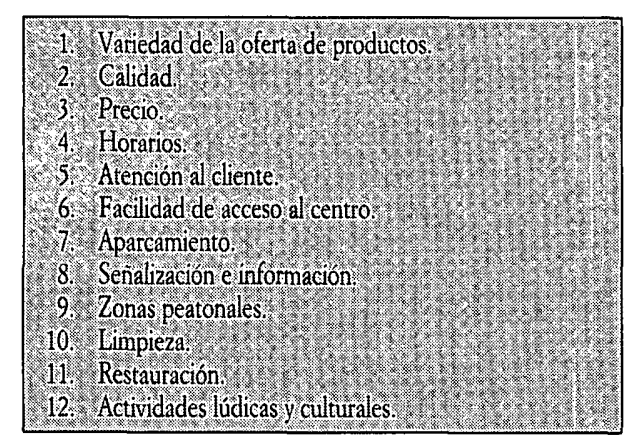

Por último, resaltar que los factores que presentan mayor diferencia entre importancia y valoración, podríamos decir los que presentan una mayor insatisfacción, son por orden de importancia: el Aparcamiento, la Limpieza y el Precio.

Desde la Diputació de Barcelona se pretende mantener anualmente la realización de estas encuestas para disponer de un indicador homogéneo de la realidad de los centros comerciales urbanos.

En el año 1999 se realizaron en seis municipios, y la media de los índices globales ponderados fue de 5,64 sobre 10 .
En el año 2000 se han realizado en estos seis municipios la misma encuesta obteniéndose una media de los índices globales ponderados de 5,74.

También en el año 2000 se han realizado un total de 13 encuestas en once nuevos municipios, en dos de ellos se realizaron en dos zonas comerciales diferentes de la ciudad, siendo en este caso la media de los 13 índices globales ponderados de 5,55.

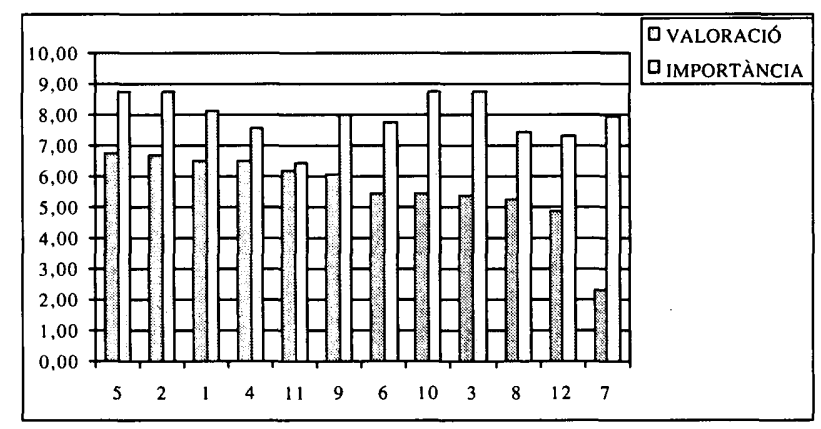

b) De sensibilización: Seminarios de experiencias de dinamización comercial. Son nueve sesiones, organizadas en todos los municipios con proyecto de dinamización, en las que los comerciantes y profesionales directamente implicados exponen experiencias exitosas de dinamización comercial. (Véase despiece adjunto.)

\section{Seminarios de experiencias de dinamización de Centros Comerciales Urbanos}

En los municipios en los que se pone en marcha un proyecto de dinamización del Centro Comercial Urbano, la Diputació de Barcelona ofrece un producto de sensibilización a los comerciantes, enfocado a presentarles cuáles son las claves del éxito de un proyecto de dinamización, qué aspectos han sido determinantes para la evolución positiva en cada uno de ellos, qué papel han jugado los diferentes interlocutores que inciden en un proyecto -comerciantes, asociaciones existentes, gerente, Ayuntamiento Dirección General de Comercio, Cámaras de Comercio, entidades bancarias-y también aspectos técnicos relacionados con el funcionamiento de los centros urbanos, como urbanismo comercial, movilidad o accesibilidad.

Pero, al mismo tiempo, los seminarios se han diseñado como un instrumento del proyecto de dinamización que facilite al gerente del proyecto la relación con el colectivo de comerciantes, que permita identificar los comerciantes que asumen con más facilidad los presupuestos que incorpora el proyecto de dinamización y los comerciantes con mayor disponibilidad para liderarlo.

Los seminarios se han diseñado como un ciclo de 9 sesiones, de unas dos horas como máximo, realizadas semanalmente el mismo día, el mediodía o por la noche, en las que participan el ponente que explica la experiencia de dinamización, el Gerente del proyecto, que ha de hacer el enlace entre la experiencia que se expone y la realidad del municipio y, por último, el moderador, que es la persona encargada de la organización del seminario, de preparar los materiales, de contactar con los diversos ponentes y sobre todo de conseguir la participación de los comerciantes en el debate posterior que se propone una vez presentada la experiencia. La idea inicial es que la presentación dure aproximadamente una hora y que el debate posterior otra hora.

Para la novena y última sesión, se propone la elaboración de un decálogo de las necesidades del proyecto de dinamización en el municipio en el que se ha realizado el seminario y se plantea como una sesión en la que se han de extraer conclusiones entre los comerciantes y los gestores del proyecto sobre la realidad del comercio del centro urbano.

Entre los ponentes destacan: gerentes de Centros Comerciales cerrados y urbanos; especialmente interesante es la información que proporciona el Gerente de un centro cerrado como L'Illa Diagonal, representantes de Juntas Directivas de Asociaciones de Comerciantes y un gestor de Mercado Municipal. 

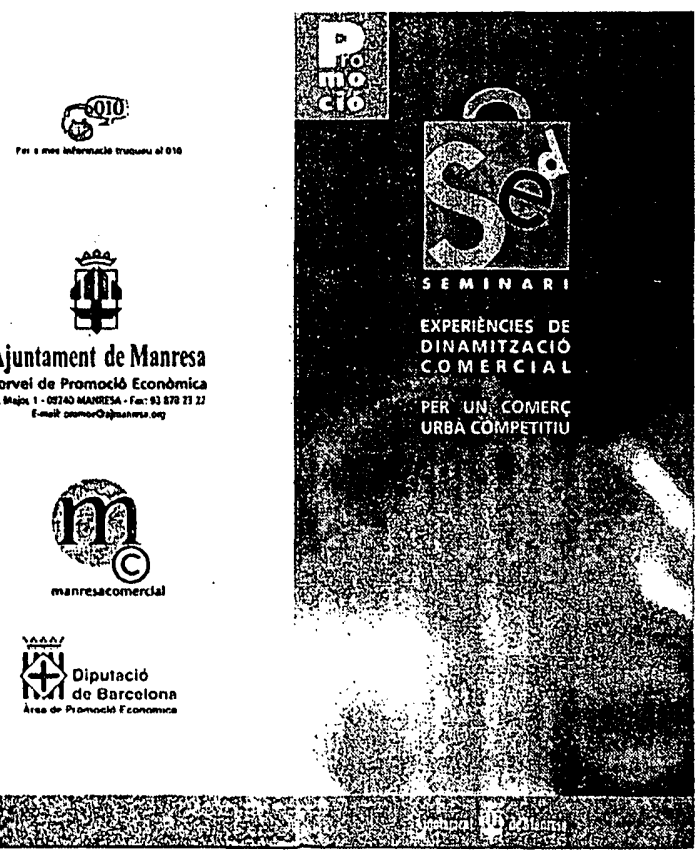

Contenido de las sesiones:

1. Los Centros Comerciales Urbanos, un objetivo para el comercio urbano: La experiencia de Granollers. Ponente: Xavier Quer (Asociación de comerciantes «Gran Centre Granollers»). El proyecto de Granollers ha sido galardonado con el Premio a la Iniciativa Comercial 1998, de la Generalitat de Catalunya, como la mejor asociación de comerciantes. Es posiblemente uno de los Centros Comerciales Urbanos más desarrollado de Cataluña.

2. ¿Pueden los comerciantes, con su esfuerzo, vencer a las Grandes Superficies Comerciales?: «El carrer de Sants». Ponente: Enric Ricart (Asociación de comerciantes «Carrer de Sants»). La calle de Sants es el centro comercial más largo de Europa y basa su competitividad en la potencia de su asociación y en las acciones de promoción conjunta.

3. Programas y experiencias de modernización comercial. Ponente: Joan Durà, empresario-comerciante del sector de la moda. Las experiencias de un empresario comercial que, mediante procesos de expansión territorial y diversificación, demuestra la capacidad del comercio detallista de adaptarse a los nuevos tiempos y a las nuevas fórmulas comerciales con éxito.

4. El comercio no se acaba en el aparador. Experiencias y modelos de diseño del entorno urbano. Ponentes: Ole Thorson (INTRA) y Guillen Díaz (D'Alpeh), expertos en urbanismo comercial. La accesibilidad, la movilidad y la coexistencia del tránsito en las zonas comerciales de nuestros municipios. Urbanismo comercial y los instrumentos necesarios para mejorar la imagen del entorno urbano del comercio. Se trata de demostrar que las necesidades urbanisticas de los comerciantes, bien canalizadas, pueden tener respuesta positiva entre los gestores del entorno urbano.

5. Asociaciones de comerciantes, algunas claves para el éxito. Ponentes: Miembros de la Junta de la Asociación de Comerciantes «Sant Feliu Centre». La asociación se constituyó en 1997 con el objetivo de dinamizar el comercio de la zona central de Sant Feliu de Llobregat. Aquel proyecto, iniciado por un grupo de comerciantes dinámicos, se ha convertido en un marco de participación y colaboración formado por la mayoría de los comercios del centro del municipio.

6. Qué acciones de animación pueden atraer clientes. Ponente: Raúl Monroy, Gerente de «L'Illa Diagonal». Uno de los principales Centros Comerciales de nueva generación basa una parte importante de su éxito en su capacidad de atracción y en la organización de actividades atractivas para unos visitantes que se convierten fácilmente en compradores.

7. La importancia de los Mercados Municipales para la activación y la dinamización comercial. Ponente: Josep Abad, Director del Mercado Municipal de Mollet del Vallès. La renovación de los Mercados Municipales, en el marco de la adaptación de los horarios a las necesidades de los consumidores, se convierte en un factor crítico para su futuro. La renovación en el caso de Mollet del Vallès ya se ha producido con éxito.

8. Modelos, diseño de espacios y relaciones con el entorno de los Centros Comerciales Cerrados. Ponente: Enrique Suárez, Gerente del Centro Comercial «Max Center» de l'Hospitalet de Llobregat. Situado en el centro de la ciudad, en las naves industriales de la antigua fábrica "La Farga», ejerce de locomotora para el comercio del entomo y se ha integrado perfectamente con los barrios Centre y Sant Josep (aparcamiento, accesos, etc.).

9. Sesión Final: resumen y conclusiones del seminario: El objetivo de la sesión es elaborar, conjuntamente y a partir de los resultados de las anteriores, el «Decálogo del comercio del centro urbano», base para la constitución de grupos estables de debate.

\subsection{Indicadores de seguimiento:}

a) Número de operadores participantes en campañas de promoción organizadas por el Proyecto de Dinamización.

b) Volumen de las aportaciones económicas efectuadas por los operadores en las campañas de promoción, porcentaje del total de los costes soportada.

c) Número de operadores participantes en ofertas de servicios complementarios a los clientes del Centro Comercial Urbano, fundamentalmente aparcamiento gratuito.

d) Número de horas efectivamente utilizadas en los aparcamientos del Centro Comercial Urbano, con los vales distribuidos desde el Centro Comercial Urbano.

e) Número de asociados a las organizaciones de los operadores implicados en el proyecto.

f) Cuota establecida por dichas organizaciones. La cuota y el número de asociados determinan el tipo de proyecto que pretenden impulsar los operadores comerciales del Centro Comercial Urbano.

2.6. Resultados esperados al finalizar el tercer año del proyecto:

a) Consolidar la corresponsabilización y la financiación conjunta del Centro Comercial Urbano por parte de los operadores (Comercio y otros servicios) y del Ayuntamiento (responsable del centro ciudad y de los servicios generales: limpieza, seguridad, accesibilidad, mantenimiento, vialidad).

b) Por parte de los operadores comerciales y de servicios, asunción de las funciones de: Cubrir los gastos fijos de estructura (sueldos y local), financiar íntegramente las campañas de promoción y de servicios a los clientes y captar recursos de publicidad, patrocinio y subvenciones supramunicipales.

c) Por parte del Ayuntamiento, colaborar en la financiación de los gastos de estructura, garantizar la calidad propia de un centro comercial de los servicios que presta en el Centro Urbano e incorporar elementos de proyecto de ciudad, no exclusivamente de comercio, a la actuación en el Centro Comercial Urbano.

d) Conseguir una única organización que integre a la mayoría de los operadores económicos del Centro Urbano, independientemente de la fórmula jurídica escogida.

e) Un mínimo de entre 150 y 200 asociados con unas cuotas de aproximadamente 6.000 pesetas mensuales, que representaría un presupuesto anual de 14,4 millones de pesetas.

f) Asunción por parte del Ayuntamiento de la cultura colectiva, en la que la concertación sea el método habitual para la toma de decisiones y hacer el seguimiento de las actuaciones. 


\section{El proyecto de dinamización: Mollet del Vallès. \\ Centre Comercial}

\section{Antecedentes}

a) Asociaciones de comerciantes en cada una de las calles del municipio con cuotas simbólicas, agrupadas en una Federación del Comercio del Mollet del Vallès más virtual que real. Relación con el Ayuntamiento basada en el enfrentamiento y la reivindicación.

b) Traslado del Mercado Municipal desde el casco antiguo a una zona de nuevo desarrollo urbanístico, en los límites del casco antiguo.

\section{Primeras actuaciones}

a) Definición de la zona del Centro Comercial Urbano.

b) Diseño de un logotipo y de una marca: «Mollet del Vallès. Centro Comercial».

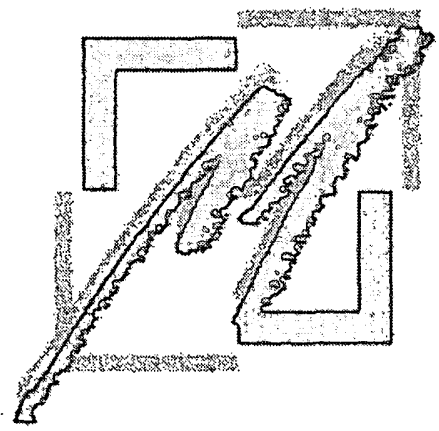

\section{Mollet del Vallès CENTRE COMERCIAL}

c) Un técnico de la empresa municipal Mercamollet, S. A., encargado de la Feria Comercial y de la gestión del Mercado Municipal, asume las funciones del Gerente del Centro Comercial Urbano.

\section{Acciones de promoción}

a) 1. ${ }^{\mathrm{a}}$ Campaña de Navidad conjunta:

a.1) Distribución de un pequeño obsequio a los clientes (una chocolatina dentro de una pequeña caja de cartón con cintas para anudarla en los paquetes y bolsas).

a.2) 170 comerciantes participantes, sobre un total de 300 en el Centro Comercial Urbano.

a.3) Se repartieron en total 27.500 obsequios.

a.4) Decoración homogénea en todo el Centro Comercial Urbano.

a.5) Aportación total de los comerciantes: 550.000 pesetas, a 20 pesetas el obsequio.

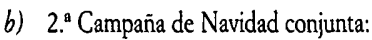

b.1) Sorteo de 26 premios de 50.000 pesetas, para consumir en los comercios participantes. Los comerciantes distribuian los boletos. Urbano.

b.3) Se repartieron en total 305.000 boletos.

b.4) Publicidad conjunta, actos de animación y fiesta final de entrega de premios con gran asistencia de público.

b.5) Aportación total de los comerciantes: 1.800 .000 pesetas, 5.500 los asociados a alguna de las asociaciones de las calles del centro y 7.000 los no asociados. La diferencia dc costes es para fomentar el ingreso en las asociaciones.

b.6) Incremento de casi el $70 \%$ en el número de comerciantes participantes y de más del $200 \%$ en las aportaciones al coste de la campaña en sólo un año.

c) Cooperación entre operadores del Centro Urbano: El comercio te invita a una Coca-Cola light:

c.1) El comercio distribuye unos tiquets que los bares y cafeterías del Centro Urbano intercambian por una Coca-Cola gratis.

c.2) Participación de 44 bares y cafeterías y de 250 comercios. c.3) La casa Coca-Cola ofrece las 20.000 botellas, que distribuye a bares y cafeterías a cambio de publicidad.

c.4) Cada comercio dispone de 80 vales por un coste de mil pesetas.

c.5) La aportación de los comerciantes, 250.000 pesetas, cubre los costes de publicidad.
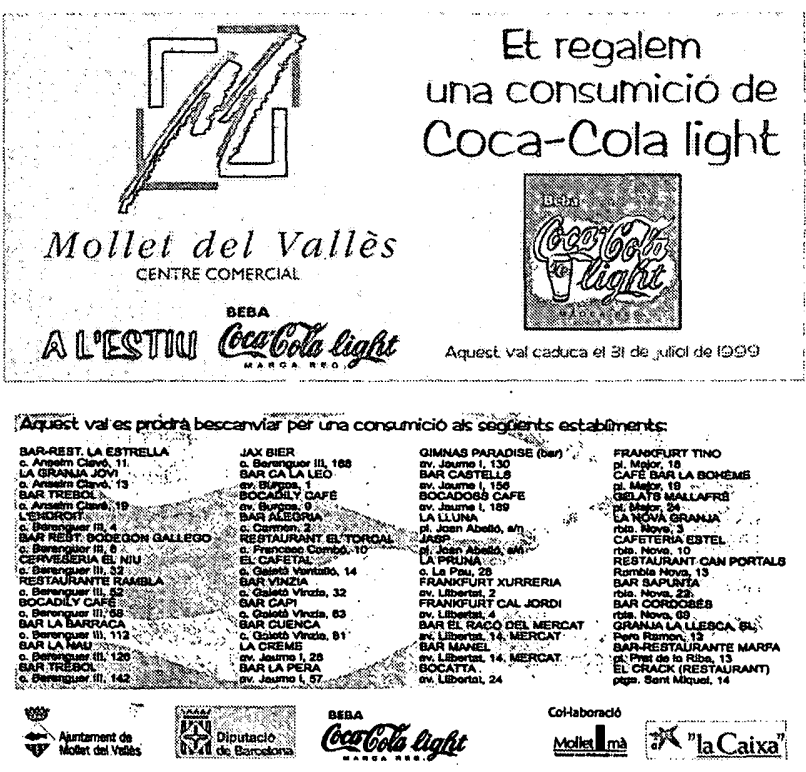

Servicios a los clientes: Creación de la tarjeta

de fidelización «Carnet del Client»
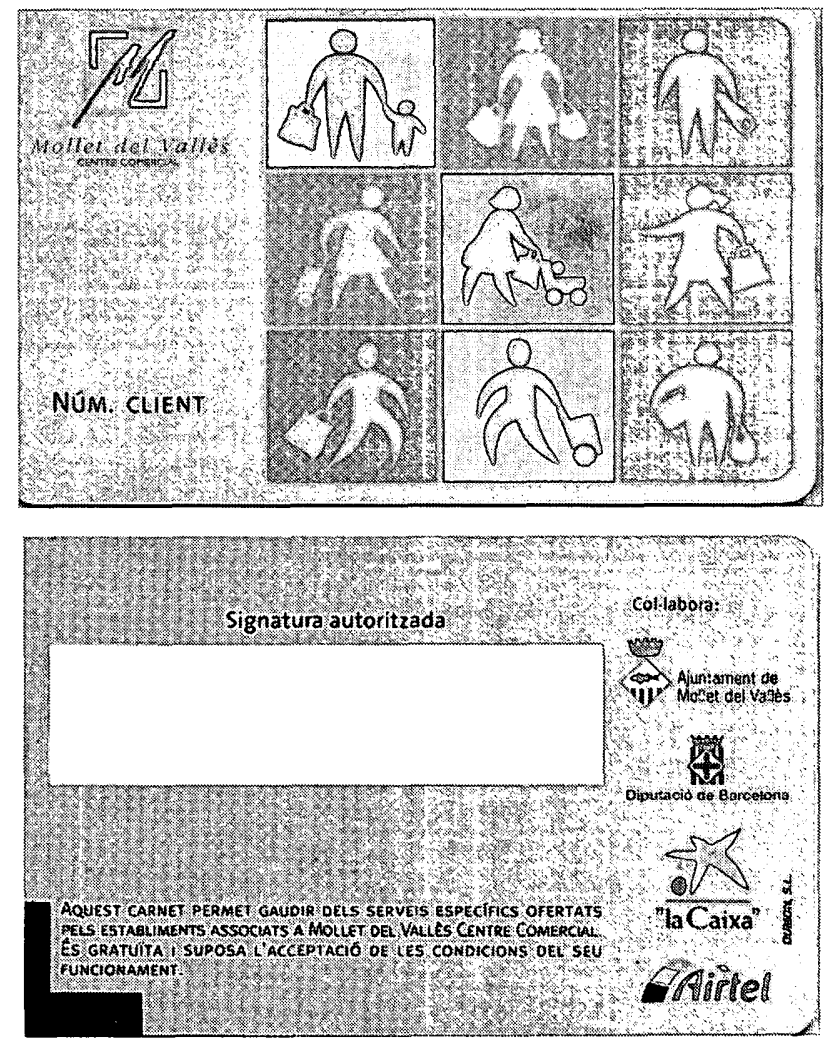

Estructura del Centro Comercial Urbano

a) Señalización comercial del Centro Urbano: OPIS, delimitación, aparcamiento.

b) Incremento del servicio de limpieza: Nuevo contrato que incluye adquisición de nuevos vehículos, incorporación de personal e incremento del número de contenedores. También una actuación específica de recogida de cartones. 
Situación de la concertación entre operadores comerciales a la finalización del proyecto Diputació-Ayuntamiento

a) Creación de una nueva asociación para todo el Centro Comercial Urbano, deno. minada «Mollet del Vallès. Centre Comercial», en noviembre del 2000

b) Contratación de un nuevo gerente del proyecto por parte de la asociación.

c) En febrero del 2001, 160 asociados, con cuotas de 3.000 pesetas al mes.

d) Establecido un marco estable de colaboración con el Ayuntamiento.

Aportaciones económicas al Proyecto

\begin{tabular}{|c|c|}
\hline \multicolumn{2}{|c|}{ 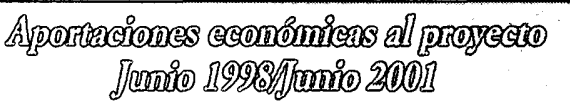 } \\
\hline & Mptas. \\
\hline $\begin{array}{l}\text { Diputació de Barcelona } \\
\text { Ajuntament de Mollet del } \\
\text { Valless }\end{array}$ & $\begin{array}{l}22,2 \\
18\end{array}$ \\
\hline $\begin{array}{l}\text { Comerciantes } \\
\text { Patrocinadores: } \\
\text { La Caixa } \\
\text { Mollet a Mà } \\
\text { Coca-Cola } \\
\text { Airtel }\end{array}$ & $\begin{array}{l}4,5 \\
5,7 \\
2,6 \\
1,6 \\
1 \\
0,5\end{array}$ \\
\hline Total & 50,4 \\
\hline
\end{tabular}

El proyecto de dinamización: Centre Vila. Per comprar i passejar. Vilafranca del Penedès

Antecedentes

a) Una única Asociación para todo el municipio, el Presidente repetía en el cargo sucesivamente. Dinámica de enfrentamiento con el Ayuntamiento por los proyectos de urbanización del Centro Urbano.

b) Elaboración de un estudio de un Plan de Dinamización, encargado por la Asociación de Comerciantes y financiado por el Ayuntamiento.

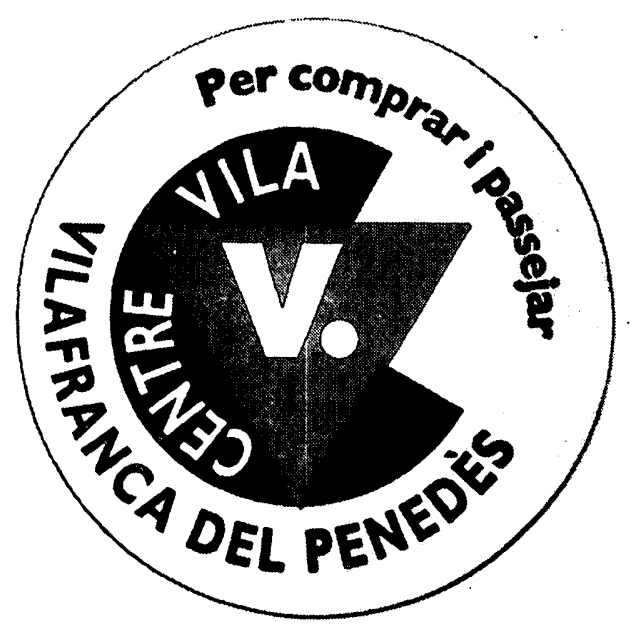

c) Apuesta del Ayuntamiento por la promoción turística de la ciudad. Existencia de un Patronato Municipal de Comercio y Turismo.

\section{Primeras actuaciones}

a) Definición de la zona del Centro Comercial Urbano

b) Diseño de un logotipo y de una marca: «Centre Vila. Per comprar i passejar».

c) Contratación de un Gerente independiente por parte del Patronato de Comercio y Turismo. d) Constitución de una nueva Asociación de Comerciantes, que adopta el nombre y la imagen del proyecto, y que abarca territorialmente la zona del Centro Comercial Urbano. Se llega a un acuerdo de federación entre la nueva asociación y la antigua, que sigue representando al conjunto del municipio.

e) Se llega a un acuerdo institucional en el que además de los comerciantes, el Ayuntamiento y la Diputació de Barcelona, también participan la Direcció General de Comerç de la Generalitat de Catalunya, la Cambra de Comerç de Barcelona y el Agrupament de Botiguers de Catalunya $(A B C)$, organización que agrupa a Asociaciones de Comerciantes de la mayoría de municipios de Catalunya.

\section{Servicios a clientes}

a) Aparcamiento gratuito.

a.1) Convenio con los cuatro aparcamientos situados en el Centre Vila, con una capacidad total de 700 plazas.

a.2) Vale gratuito, válido para los cuatro aparcamientos, por una hora, acumulativo, a cualquier hora o día de la semana, con opción a incluir fecha de caducidad solicitado por algunos comerciantes.

a.3) Los comerciantes solicitan a Centre Vila talonarios con 25 vales, pero sólo los pagan una vez han sido utilizados.

a.4) Coste de la hora para el asociado: 100 pesetas. Coste de venta al público: 195 pesetas.

a.5) Consumo medio mensual en el primer año de funcionamiento: 2.500 horas gratuitas ưilizadas.

a.6) Campaña de promoción a los comerciantes: Por la compra de dos talonarios de 25 , uno gratis. Los cinco primeros vales que se adquieren cada mes son gratuitos. Sistema de descuentos y rapels por volumen de vales adquiridos.

a.7) Campaña de promoción a los clientes: Adhesivos en los aparadores y en las cajas registradoras. Azafatas por las calles del Centre Vila distribuyendo información. Carteles informativos en los aparcamientos y cabinas telefónicas. Campañas en la prensa y radio locales.

a.8) Incorporación del lema aparcamiento gratuito en todas las acciones organizadas por Centre Vila.

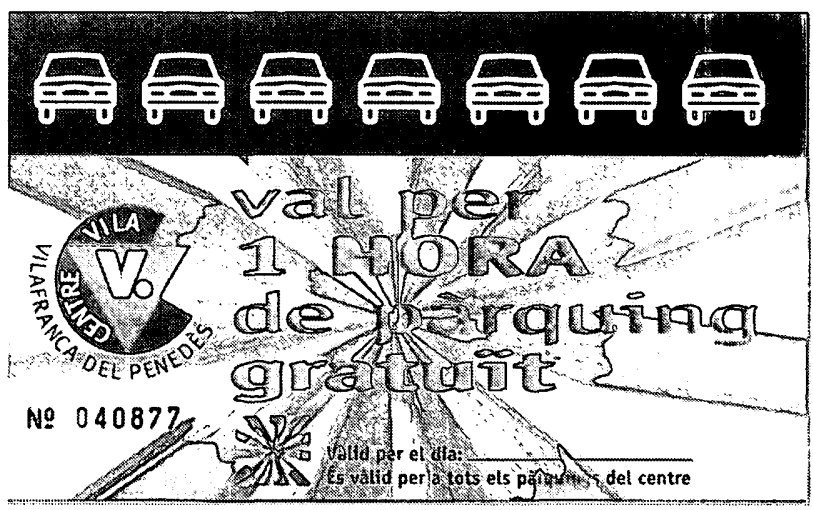

Tots els associats de Centre Vila et regalem l'aparcament.

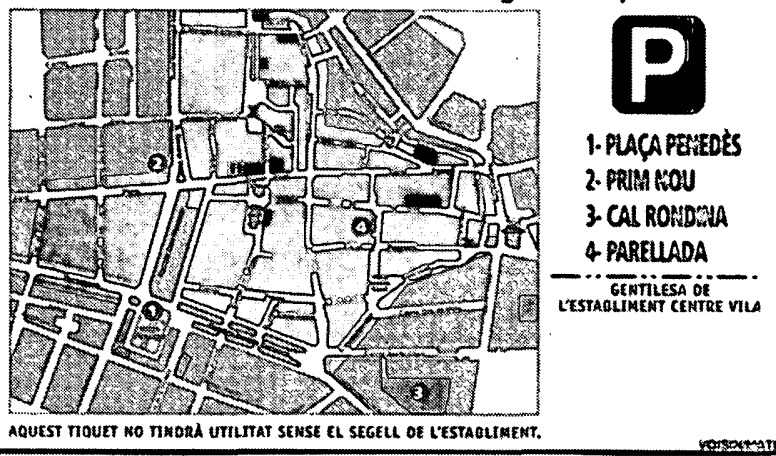

b) Experiencia piloto de servicio gratuito de guardería infantil durante la Campaña de Navidad. 


\section{Servicios a los comerciantes}

Acuerdo con la Caja de Ahorros local, que incluye rebajas en las comisiones por la utilización de tarjetas — con independencia de la entidad emisora - por parte de los clientes, datáfono gratuito, cuentas de crédito con interés preferente, mejoras en las condiciones de la cuenta corriente y ventajas para los empleados.

\section{Situación de la concertación entre operadores} comerciales a la finalización del proyecto con financiación institucional

a) Gerente contratado por la propia asociación desde hace más de un año.

b) En febrero de 2001, 275 asociados, sobre un total de 300, con cuotas mensuales de 6.000 pesetas.

c) Marco estable de colaboración con el Ayuntamiento, con la creación de comisiones para seguimiento de temas concretos, cómo la reforma urbanística, la limpieza, la seguridad o la recogida de cartones de los comercios.

\section{Aportaciones económicas al Proyecto}

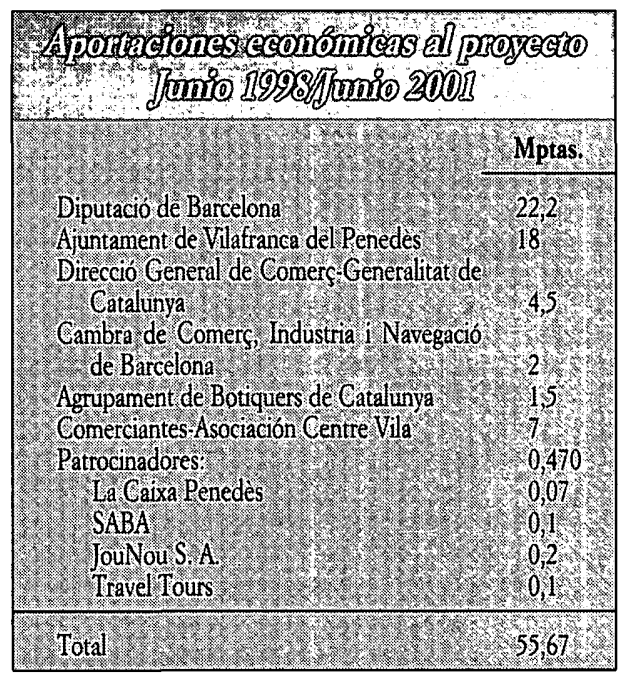

* Economista y analista económico de La Vanguardia. Responsable de Comercio y estrategias territoriales de la Diputació de Barcelona.

** Director del Proyecto de Dinamización de Centros Comerciales Urbanos de la Diputació de Barcelona.
1 Quaderns de promoció econòmica local, 1, La dinamització dels centres comercials, Diputació de Barcelona, noviembre 1999. 